\title{
Application of the Turbulence Models at Low Reynolds Number for Horizontal-Axis Wind Turbine Design by using Computational Fluid Dynamics (CFD)
}

\author{
M. H. Ouahabi ${ }^{a^{*}}$, M. M. Ichenial ${ }^{b}$, A. El Hajjaji ${ }^{b}$ and F. Benabdelouahab ${ }^{a}$ \\ ${ }^{a}$ Department of Physics, Laboratory of Condensed Matter and Radiation, \\ Faculty of Sciences Tetouan, Abdelmalek Essaadi University, 93030, Morocco \\ ${ }^{b}$ Department of Physics, Faculty of Sciences at Tetouan, Abdelmalek Essaadi \\ University, 93030, Morocco \\ *Corresponding author: Email: ouahabitim.gc@gmail.com
}

\begin{abstract}
In the present paper, the aerodynamic analysis of two dimensional model of NACA 0012 airfoil is presented to improve their aerodynamic characteristics such us lift, drag coefficients and the lift to drag ratio for shape design of Horizontal-Axis Wind Turbine (HAWT). The Reynolds-averaged Navier Stokes (RANS) in conjunction with three turbulence models, namely, the Spalart Allmaras, the Transition kkL-omega and the k- $\omega$ shear stress transport (SST) are solved by using the commercial code ANSYS-FLUENT, a widely used finite-element method (FEM) based software in computational fluid dynamics (CFD). The computational domain was structured with ' $C$ ' topology block-structured mesh and the steady solver was used to capture the dynamic stall phenomena. Both grid and time step were optimized to reach independent solutions. Particularly a high quality $2 D$ mesh was obtained using the ANSYS Meshing tool. Spatial discretization algorithm, interpolation scheme, pressure - velocity coupling and turbulence boundary condition were also optimized. The $2 D$ CFD model was calibrated and validated comparing the numerical results with experimental data, available in scientific literature from National Advisory Committee for Aeronautics (NACA). As a result, a good agreement between numerical and experimental data was found. Then the blade element momentum method (BEM) which divides the blade into several blade elements used to calculate ideal geometry for a turbine blade. The present work represents the basis to develop an accurate three dimensional Horizontal-Axis Wind Turbine (HAWT) model and may be used to support wind tunnel experiments.
\end{abstract}

Keywords: Computational fluid dynamics (CFD), airfoil, aerodynamic coefficients, drag coefficient, lift coefficient

\section{Introduction}

In wind industry, in the recent years the aerodynamic and structural optimization of wind turbines and the optimization of wind farm layouts for maximum power production with minimum land use have been the subject of great deal of research [1]. For wind turbines, one of the most important design criteria is to maximize the harvested wind energy while satisfying other conditions such as structural integrity, economics, geography, etc. Therefore, wind turbine design is a multidisciplinary design process. Meanwhile, for more cost reduction considerations there are three approaches are available to analyze the flow around an airfoil section of Horizontal-Axis Wind Turbine. Firstly, field or wind tunnel testing provides accurate results but is highly complex, expensive and cannot be applied for the large wind turbines available today. Secondly, analytical and semi-empirical models exist, which adopt simplifying assumptions and are 
thus not universally reliable. Thirdly, the computational fluid dynamics, also known as "CFD", has been driven by the need for faster and more accurate methods for the calculations of flow fields around configurations of technical interest and it offers the best support or alternative to direct measurements [2]. There are many commercial CFD packages available for modeling flow in or around objects. The computer simulations show features and details that are difficult, expensive or impossible to measure or visualize experimentally. For this reason, accurate Computational Fluid Dynamics (CFD) methods are necessary and several works documenting the applications of CFD for wind turbines appear frequently in literature [3]. The calculations were made by solving the incompressible Navier-Stokes equations considering the flow as steady. The turbulent flow was calculated using the Spalart-Allmaras, Transition kkL-omega, k-omega SST turbulence models $[4,6]$. We used 2D calculations to check the ability to simulate the performance of the airfoil used on the wind turbine. The results were verified by comparing them to measurements data from the National Advisory Committee for Aeronautics (NACA) in the wind tunnel. Finally, some preliminary investigation of shape optimization of 3D wind turbine blades at low Reynolds numbers is carried out. The optimization employs a 3D straight untapered wind turbine blade with cross section of NACA 0012 airfoils as the geometry of baseline blade. The optimization objective is to achieve maximum $C_{L} / C_{D}$ as well as maximum $C_{L}$. The multi-objective genetic algorithm is employed together with the commercially available software FLUENT for calculation of the flow field using the Reynolds-Averaged Navier-Stokes (RANS) equations in conjunction with a one-equation Spalart-Allmaras turbulence model. The results show excellent performance of the optimized wind turbine blade and indicate the feasibility of optimization on real wind turbine blades with more complex shapes in the future.

\section{Experimental Methods}

The boundary layers in general are two types, namely, laminar and turbulent. The flow in laminar layer is smooth and free from any eddying motion. While the flow in the turbulent layer is characterized by the presence of a large number of relatively small eddies [7]. Because the eddies in the turbulent produce a transfer of momentum from the relatively fast-moving outer parts of the boundary layer to the portions closer to the surface, the distribution of average velocity is characterized by relatively higher velocities near the surface and a greater total boundary-layer thickness in a turbulent boundary layer than in a laminar boundary layer developed under otherwise identical conditions [8]. Skin friction is therefore higher for turbulent boundary-layer flow than for laminar flow. Generally, the flow of fluid can be mathematically described by the equations for conservation of mass, momentum and energy. These partial differential equations (PDEs) are based on the assumption that the fluid can be described as a continuous medium. The techniques used to solve the problem replace the PDEs by a set of algebraic equations by dividing the physical domain into a large number of discrete control volumes, called elements or cells. Within these cells algebraic relationships describe how the flow variables, such as velocity, temperature or pressure, vary locally with the space coordinates. This is the general idea behind Computational Fluid Dynamics (CFD). The continuity and momentum equations are combined with one of the following turbulence models which are briefly presented as follows.

\subsection{The Spalart-Allmaras}

The Spalart and Allmaras (1992) model is a relatively simple one-equation model that solves a modeled transport equation for the kinematic eddy (turbulent) viscosity. It was designed specifically for aerospace applications involving wall-bounded flows and has been shown to give good results for boundary layers subjected to adverse pressure gradients. It is also gaining popularity for turbo machinery applications. In its original 
form, it is effectively a low-Reynolds number model, requiring the viscous-affected region of the boundary layer to be properly resolved. The near-wall gradients of the transported variable in the model are much smaller than the gradients of the transported variables in the $\mathrm{k}-\omega$ or $\mathrm{k}-\varepsilon$ models. In the turbulence model of Spalart-Allmaras, the transport equation can be written as:

$$
\frac{\mathrm{D} v}{\mathrm{Dt}}=\mathrm{c}_{\mathrm{bl}}\left(1-\mathrm{f}_{\mathrm{t} 2}\right) \tilde{\mathrm{S}} v+\frac{1}{\sigma}\left[\nabla \cdot((v+v)) \nabla v+\mathrm{c}_{\mathrm{b} 2}(\nabla v)^{2}\right]-\left(\mathrm{c}_{\mathrm{w} 1} \mathrm{f}_{\mathrm{w}}-\frac{\mathrm{c}_{\mathrm{bl}}}{\kappa^{2}} \mathrm{f}_{\mathrm{t} 2}\right)\left(\frac{v}{\mathrm{~d}}\right)^{2}+\mathrm{f}_{\mathrm{t} 1} \Delta \mathrm{U}^{2}
$$

The form of the operating parameters $v$ as below. Where $v$ is the molecular viscosity calculated by the Sutherland's (1893) law. The four terms on the right hand side correspond to production, diffusion, dissipation and transition, respectively. The individual components of the production term are defined as:

$$
\begin{aligned}
& \tilde{\mathrm{S}}=\mathrm{S}+\frac{v}{\kappa^{2} \mathrm{~d}^{2}}\left\{\left[1-(v / v)\left[1+\frac{(v / v)^{4}}{\left[(v / v)^{3}+\mathrm{C}_{\mathrm{w} 3}^{6}\right]}\right]^{6}+\mathrm{C}_{\mathrm{w} 3}^{6}\right]\right\}^{-1 / 6} \\
& \mathrm{f}_{\mathrm{w}}=\frac{v}{\tilde{\mathrm{S}}^{2} \mathrm{~d}^{2}}\left[1+\mathrm{C}_{\mathrm{w} 2}\left(\left(\frac{v}{\tilde{\tilde{S}^{2} \mathrm{~d}^{2}}}\right)^{5}-1\right)\right]\left(1+\mathrm{C}_{\mathrm{w} 3}^{6}\right)^{1 / 6}\left\{\left[1+\mathrm{C}_{\mathrm{w} 2}\left(\left(\left(\frac{\bar{v}}{\overline{\mathrm{Sk}^{2} \mathrm{~d}^{2}}}\right)^{5}-1\right)\right)\right]^{6}+\mathrm{C}_{\mathrm{w} 3}^{6}\right\}^{-1 / 6} \\
& \mathrm{f}_{\mathrm{t} 1}=\mathrm{C}_{\mathrm{t} 1} \mathrm{~g}_{\mathrm{t}} \exp \left[-\mathrm{C}_{\mathrm{t} 2} \frac{\omega_{\mathrm{t}}^{2}}{\Delta \mathrm{U}^{2}}\left(\mathrm{~d}^{2}+\mathrm{g}_{\mathrm{t}}^{2} \mathrm{~d}_{\mathrm{t}}^{2}\right)\right] \\
& \mathrm{f}_{\mathrm{t} 2}=\mathrm{C}_{\mathrm{t} 3} \exp \left[-\mathrm{C}_{\mathrm{t} 4}(\bar{v} / v)^{2}\right]
\end{aligned}
$$

Where $\mathrm{S}$ is the magnitude of the vorticity, $\mathrm{d}$ is the distance to the closest wall, $\mathrm{d}_{\mathrm{t}}$ is the distance from the point in the flow field to the trip on the wall, is the wall vorticity at the trip, $\Delta \mathrm{U}$ is the difference between velocity at the field point and that at the trip, where $\mathrm{g}_{\mathrm{t}}=\min \left(0.1 \Delta \mathrm{U} / \omega_{\mathrm{t}} \Delta \mathrm{x}_{\mathrm{t}}\right)$ is the grid spacing along the wall at the trip. The empirical constants of the Spalart-Allmaras model are:

\begin{tabular}{cccccccccccc}
$\mathrm{C}_{\mathrm{b} 1}$ & $\sigma$ & $\mathrm{C}_{\mathrm{b} 2}$ & $\kappa$ & $\mathrm{C}_{\mathrm{w} 1}$ & $\mathrm{C}_{\mathrm{w} 2}$ & $\mathrm{C}_{\mathrm{w} 3}$ & $\mathrm{C}_{\mathrm{v} 1}$ & $\mathrm{C}_{\mathrm{t} 1}$ & $\mathrm{C}_{\mathrm{t} 2}$ & $\mathrm{C}_{\mathrm{t} 3}$ & $\mathrm{C}_{\mathrm{t} 4}$ \\
\hline 0.66 & 0.1355 & 0.4187 & 3.239 & 0.3 & 2.0 & 7.1 & 1 & 1 & 1.2 & 0.5 \\
\hline
\end{tabular}

\subsection{The Transition kkL-omega}

The validated model which called kkL-omega transition model code is based on Walters and Cokkjat's [8] work. In this model, three additional transport equations of turbulent kinetic energy $k_{T}$, laminar kinetic energy $k_{L}$ and the specific dissipation $\omega$ are listed as follows:

$$
\frac{\mathrm{Dk}_{\mathrm{T}}}{\mathrm{Dt}}=\mathrm{P}_{\mathrm{kT}}+\mathrm{R}_{\mathrm{BP}}+\mathrm{R}_{\mathrm{NAT}}+\omega \kappa_{\mathrm{T}}-\mathrm{D}_{\mathrm{T}}+\frac{\partial}{\partial \mathrm{x}_{\mathrm{j}}}\left[\left(v+\frac{\alpha_{\mathrm{T}}}{\sigma_{\mathrm{K}}}\right) \frac{\partial \kappa_{\mathrm{T}}}{\partial \mathrm{x}_{\mathrm{j}}}\right]
$$




$$
\begin{aligned}
& \frac{D k_{L}}{D t}=P_{k L}+R_{B P}+R_{N A T}+D_{L}+\frac{\partial}{\partial x_{j}}\left[v \frac{\partial \kappa_{L}}{\partial x_{j}}\right] \\
& \frac{D \omega}{D t}=C_{\omega 1} \frac{\omega}{K_{T}} P_{K T}+\left(\frac{C_{\omega R}}{f_{W}}-1\right) \frac{\omega}{K_{T}}\left(R_{B P}+R_{N A T}\right)-C_{\omega 2} \omega^{2}+C_{\omega 3} f_{\omega} \alpha_{T} f_{W}{ }^{2} \frac{\sqrt{\kappa_{\mathrm{T}}}}{d^{3}}+\frac{\partial}{\partial x_{j}}\left[\left(v+\frac{\alpha_{\mathrm{T}}}{\sigma_{\mathrm{K}}}\right) \frac{\partial \omega}{\partial x_{j}}\right]
\end{aligned}
$$

More detail mechanism explanation about this transition model can be found in [6] and [7].

\subsection{The k-omega SST turbulence model}

The SST $k-\omega$ turbulence model [9] is a two equation eddy-viscosity model which has become very popular. The shear stress transport (SST) formulation combines the better of two worlds. The use of a $\mathrm{k}-\omega$ formulation in the inner parts of the boundary layer makes the model directly usable all the way down to the wall through the viscous sub-layer. Hence the SST $\mathrm{k}-\omega$ model can be used as a Low-Reynolds turbulence model without any extra damping functions. The SST formulation also switches to a $\mathrm{k}-\varepsilon$ behavior in the free-stream and thereby avoids the common $\mathrm{k}-\omega$ problem that the model is too sensitive to the inlet free-stream turbulence properties. The authors who use the SST $\mathrm{k}-\omega$ model often merit it for its good behavior in adverse pressure gradients and separating flow. The SST $\mathrm{k}-\omega$ model does produce a bit too large turbulence levels in regions with large normal strain, like stagnation regions and regions with strong acceleration. The SST $\mathrm{k}-\omega$ turbulence model is governed by:

$$
\begin{aligned}
& \frac{\mathrm{D} \rho \mathrm{k}}{\mathrm{Dt}}=\tau_{\mathrm{ij}} \frac{\partial \mathrm{u}_{\mathrm{i}}}{\partial \mathrm{x}_{\mathrm{j}}}+\beta^{*} \rho \omega \mathrm{k}+\frac{\partial}{\partial \mathrm{x}_{\mathrm{j}}}\left[\left(\mu+\sigma_{\mathrm{k}} \mu_{\mathrm{t}}\right) \frac{\partial \mathrm{k}}{\partial \mathrm{x}_{\mathrm{j}}}\right] \\
& \frac{\mathrm{D} \rho \omega}{\mathrm{Dt}}=\frac{\gamma}{v_{\mathrm{t}}} \tau_{\mathrm{ij}} \frac{\partial \mathrm{u}_{\mathrm{i}}}{\partial \mathrm{x}_{\mathrm{j}}}-\beta \rho \omega^{2}+\frac{\partial}{\partial \mathrm{x}_{\mathrm{j}}}\left[\left(\mu+\sigma_{\mathrm{k}} \mu_{\mathrm{t}}\right) \frac{\partial \omega}{\partial \mathrm{x}_{\mathrm{j}}}\right]+2 \rho\left(1-\mathrm{F}_{1}\right) \sigma_{\omega} \frac{1}{\omega} \frac{\partial \mathrm{k}}{\partial \mathrm{x}_{\mathrm{j}}} \frac{\partial \omega}{\partial \mathrm{x}_{\mathrm{j}}}
\end{aligned}
$$

Where $\beta^{*}=\varepsilon / \mathrm{k} \omega$ and the turbulence stress tensor is

$$
\tau_{\mathrm{ij}}=-\rho \overline{\mathrm{u}_{\mathrm{i}}^{\prime} \mathrm{u}_{\mathrm{j}}^{\prime}}=\mu_{\mathrm{t}}\left(\frac{\partial \mathrm{u}_{\mathrm{i}}}{\partial \mathrm{x}_{\mathrm{j}}}+\frac{\partial \mathrm{u}_{\mathrm{j}}}{\partial \mathrm{x}_{\mathrm{i}}}-\frac{2}{3} \frac{\partial \mathrm{u}_{\mathrm{k}}}{\partial \mathrm{x}_{\mathrm{k}}} \delta_{\mathrm{ij}}\right)-\frac{2}{3} \rho \mathrm{k} \delta_{\mathrm{ij}}
$$

The turbulence viscosity can be estimated by $v_{\mathrm{t}}=\mathrm{a}_{1} \mathrm{k} / \max \left(\mathrm{a}_{1} \mathrm{k}, \Omega \mathrm{F}_{2}\right)$, where $\Omega$ is the absolute value of the vorticity, and the function $F_{2}$ is given by:

$$
\mathrm{F}_{2}=\tanh \left\{\max \left(\frac{2 \sqrt{\mathrm{k}}}{0.09 \omega \mathrm{y}} \frac{500 v}{\mathrm{y}^{2} \omega}\right)\right\}^{2}
$$

Where $y$ is the distance to the nearest surface. The coefficients $\beta, \gamma, \sigma_{\mathrm{k}}$ and $\sigma_{\omega}$ are defined as functions of the coefficients of the $k-\omega$ and $k-\varepsilon$ turbulence models and they are listed as follows:

$$
\begin{gathered}
\beta=F_{1} \beta_{1}+\left(1-F_{1}\right) \beta_{2}, \gamma=F_{1} \gamma_{1}+\left(1-F_{1}\right) \gamma_{2} \\
\sigma_{k}=F_{1} \sigma_{k 1}+\left(1-F_{1}\right) \sigma_{k 2}, \sigma_{\omega}=F_{1} \sigma_{\omega 1}+\left(1-F_{1}\right) \gamma_{2} \sigma_{\omega 2}
\end{gathered}
$$

Where $\mathrm{F}_{1}$ function is:

$$
\mathrm{F}_{1}=\tanh \left\{\left[\min \left[\max \left(\frac{\sqrt{\mathrm{k}}}{0.09 \omega \mathrm{y}}\right), \frac{4 \rho \sigma_{\omega 2} \mathrm{k}}{\mathrm{CD}_{\mathrm{k} \omega} \mathrm{y}^{2}}\right]\right]^{4}\right\}
$$


The coefficient $\mathrm{CD}_{\mathrm{k} \omega}$ is:

$$
\mathrm{CD}_{\mathrm{k} \omega}=\max \left(2 \rho \sigma_{\omega 2} \frac{1}{\omega} \frac{\partial \mathrm{k}}{\partial \mathrm{x}_{\mathrm{j}}} \frac{\partial \omega}{\partial \mathrm{x}_{\mathrm{j}}}, 10^{-20}\right)
$$

The empirical constants of the $\mathrm{k}-\omega$ SST model are as follows:

\begin{tabular}{ccccccccc}
$\beta^{*}$ & $\beta_{1}$ & $\beta_{2}$ & $\gamma_{1}$ & $\gamma_{2}$ & $\sigma_{\mathrm{k} 1}$ & $\sigma_{\mathrm{k} 2}$ & $\sigma_{\omega 1}$ & $\sigma_{\omega 2}$ \\
\cline { 2 - 8 } 0.09 & 0.075 & 0.075 & 0.5532 & 0.4404 & 0.85 & 1.0 & 0.5 & 0.856 \\
\hline
\end{tabular}

\section{Computational Methodology}

We need to set certain boundary condition in the inlet, outlet, velocity magnitude and direction in order to create simulation. The analysis was carried out at a Reynolds numbers equal to $\mathrm{Re}=5.10^{4}, \mathrm{Re}=1.10^{5}, 2.10^{5}$ and $\mathrm{Re}=1.10^{6}$, we define the velocity at the inlet according to our Reynolds numbers, and the chord length of the airfoil is $1 \mathrm{~m}$. We assume the gage pressure at the outlet is also Zero in order to validate the present simulation. The free stream temperature is $300 \mathrm{~K}$, which is the same as the environmental temperature. The density of the air at the given temperature is $\sigma=1,225 \mathrm{~kg} / \mathrm{m}^{3}$ and the viscosity is $\mu=1.7894 .10^{-4}$. For these Reynolds numbers, the flow can be described as incompressible. This is an assumption close to reality and it is not necessary to resolve the energy equation. A segregated, implicit solver was utilized (ANSYS Fluent 16.2, 2014). Calculations were done for angles of attack ranging from $-20^{\circ}$ to $20^{\circ}$. The airfoil profile, boundary conditions and meshes were all created in the ICEM CFD 16.2. A grid generation system has been developed that enable grid generation for complex configuration. The system called ICEM/CFD is described and its role in computational fluid dynamics (CFD) applications is presented. The capabilities of the system include fall computer aided design (CAD), grid generation on the actual geometry definition using robust surface projection algorithms, interfacing easily with knows CAD packages through common file formats for geometry transfer, grid quality evaluation of the volume grid, coupling boundary condition set-up for block faces with grid topology generation, multi-block grid generating with or without point continuity and block to block interface requirement, and generating grid files directly compatible with known flow solvers [10]. The interactive and integrated approach to the problem of computational grid generation not only substantially reduces more power time but also increases the flexibility of later grid modification and enhancements. The computational grid used in the present study is a ' $\mathrm{C}$ ' topology block-structured grid generated for 2D NACA 0012 airfoil. In order to include the transition affects in the aerodynamic coefficients calculation and get accurate results for the drag coefficient. The transition point from laminar to turbulent flow on the airfoil was determined about 0.25 from leading edge (upper and lower) and the computational mesh was split in two regions, a laminar and a turbulent region. The transition location on the upper and lower surface predicted by the kkL-omega transition model. Respectively, if the simulation result was larger than the experimental data it meant that the simulated turbulent zone was larger than the real and a new value for the transition point had to be chosen, righter than the initial one.

\subsection{Lift, Drag and Non-dimensional Parameters}

On an airfoil, the resultants of the forces are usually resolved into two forces and one moment. The component of the net force acting normal to the incoming flow stream is known as the lift force and the component of the net force acting parallel to the incoming flow stream is known as the drag force. Lift and drag coefficients $C_{L}$ and $C_{D}$ are defined 
as:

$$
\begin{aligned}
& \mathrm{c}_{\mathrm{L}}=\frac{\mathrm{L}}{\frac{1}{2} \rho \mathrm{cU_{ \infty } ^ { 2 }}} \\
& \mathrm{c}_{\mathrm{D}}=\frac{\mathrm{D}}{\frac{1}{2} \rho \mathrm{c} \mathrm{U}_{\infty}^{2}} \\
& \mathrm{c}_{\mathrm{p}}=\frac{\mathrm{p}-\mathrm{p}_{\infty}}{\frac{1}{2} \rho \mathrm{U}^{2}}
\end{aligned}
$$

Where: $\rho$ is the air density and $\mathrm{c}$ the length of the airfoil, often denoted by the chord. Note that the unit for the lift and drag in equations (17) and (18) is force per length (in $\mathrm{N} / \mathrm{m}$ ). A chord line can be defined as the line from the trailing edge to the nose of the airfoil.

\subsection{Blade Element Momentum (BEM) Theory}

The blade element momentum (BEM) theory is a compilation of both momentum theory and blade element theory [11]. Momentum theory, which is useful in predicted ideal efficiency and flow velocity, is the determination of forces acting on the rotor to produce the motion of the fluid. Theory determines the forces on the blade as a result of the motion of the fluid in terms of the blade geometry, by combining the two theories, BEM theory, also known as strip theory, and relates rotor performance to rotor geometry.

\section{Results and Discussion}

The process of CFD simulation begins with the creation of a two and three dimensional domain and its proper discretization. The aerodynamic characteristics of NACA 0012 for various Reynolds numbers were done in order to be able to compare the results from the different turbulence models and then validate them with existing experimental data from National Advisory Committee for Aeronautics (NACA). To do that, the model was solved for a set of angles of attack from -10 to $10^{\circ},-15^{\circ}$ to $15^{\circ}$ and $-20^{\circ}$ to $20^{\circ}$. For every angle we let the adaptive simulation runs to produce a sequence of outputs, where we expect to go from low lift through a linear growth of the lift coefficient and growth of the drag coefficient to stall, where lift decreases and drag increases dramatically. Figures 1 to 4 show the lift and drag coefficients which are plotted as functions of the angle of attack, while the drag coefficient plotted as a function of lift coefficient. Some variation of this format occur, mainly in regard to the plotting of the drag and lift data.

a

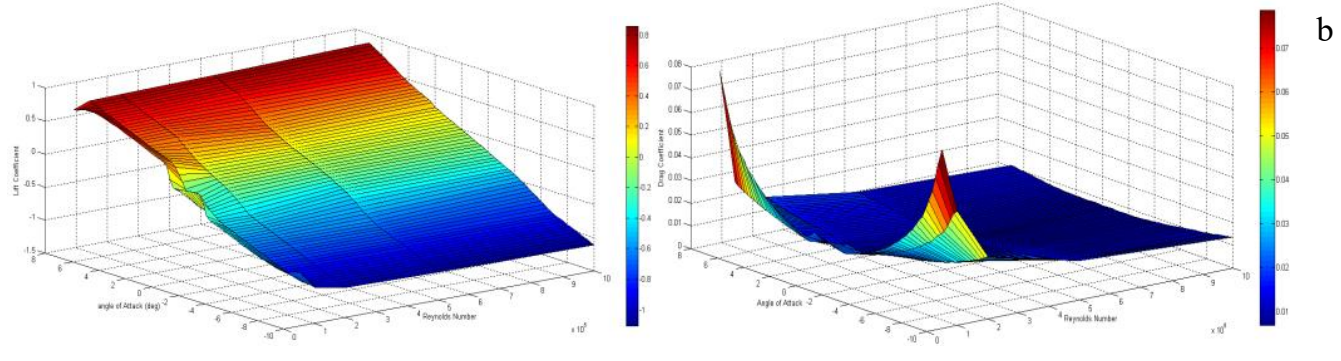

Figure 1. (a) Lift Coefficients Variations vs Angle of Attack by using the Numerical Data, (b) Drag Coefficients Variations vs Angle of Attack by using the Numerical Data 


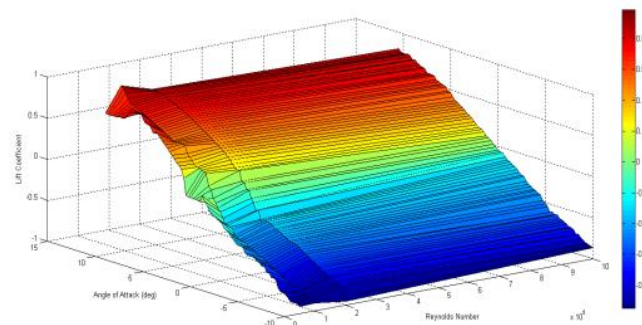

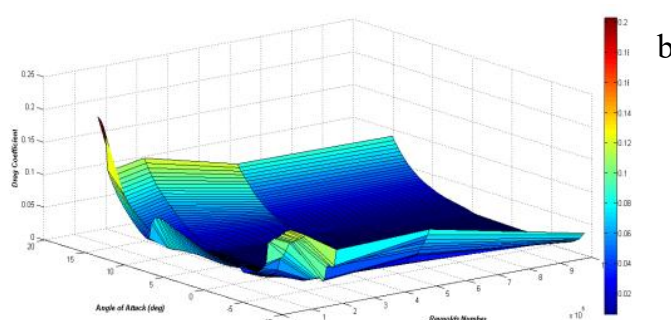

Figure 2. (a) Lift Coefficients Variations vs Angle of Attack by using k-omega SST Turbulence Model, (b) Drag Coefficients Variations vs Angle of Attack by using k-omega SST Turbulence Model

a
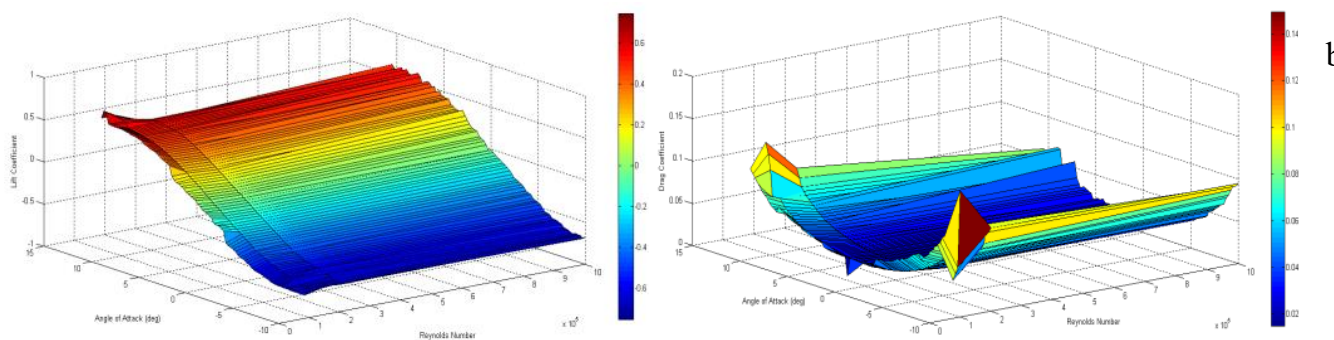

Figure 3. (a) Lift Coefficients Variations vs Angle of Attack by using Transition kkL-omega Turbulence Model, (b) Drag Coefficients Variations vs Angle of Attack by using Transition kkL-omega Turbulence Model
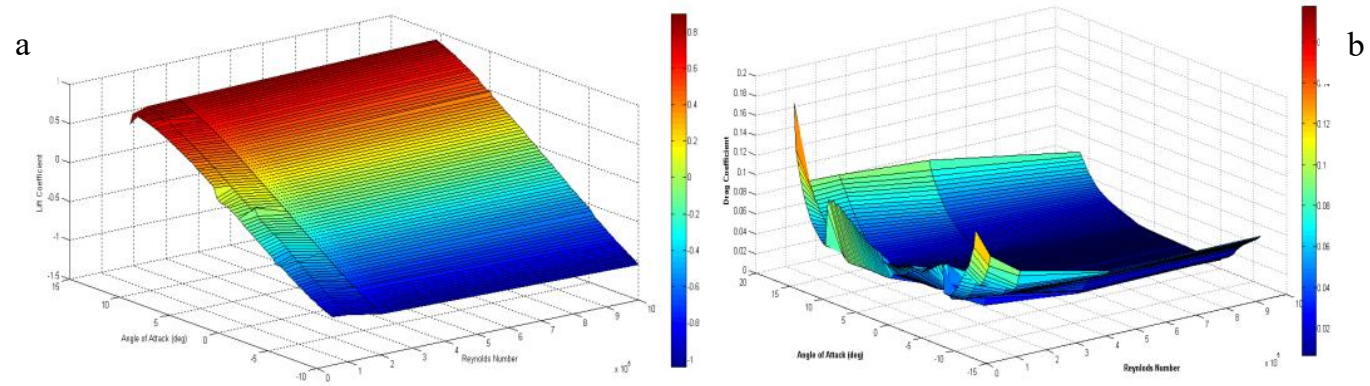

Figure 4. (a) Lift Coefficients Variations vs Angle of Attack by using Spalart-Allmaras Turbulence Model, (b) Drag Coefficients Variations vs Angle of Attack by using Spalart-Allmaras Turbulence Model

To retain consistency with the tabular data, all performance coefficients in this work are plotted as a function of angle of attack. The coefficient behavior show in figures is typical. There is a range of angle of attack where the lift coefficient varies linearly at some points, a maximum value of lift coefficient is reached where a further increase in angle of attack causes a decrease in lift. This is referred to as stall and the reasons for it are discussed later. There is a region of lift coefficient where the drag coefficient has its lowest values. Outside this range, it increases, again sometimes rapidly. This increase is related to the stall which also causes the loss in lift. All three models had a good agreement with the experimental data at angles of attack from -5 to $5^{\circ}$ and the same behavior at all angles of attack until stall, these are illustrated in Figures from 1 to 4 . It was also observed that as the Reynolds number increased the drag coefficient decreased. When the boundary layer was fully turbulent the reduction of was more intense and when there was a transition from laminar to turbulent, was reduced to a much lower rate. Also it 
was obvious that the Spalart-Allmaras turbulence model had the same behavior with the experimental data as well as after stall angle. The upper surface of the airfoil experienced a higher velocity compared to the lower surface. That was expected from the pressure distribution. As the angle of attack increased the upper surface velocity was much higher than the velocity of the lower surface

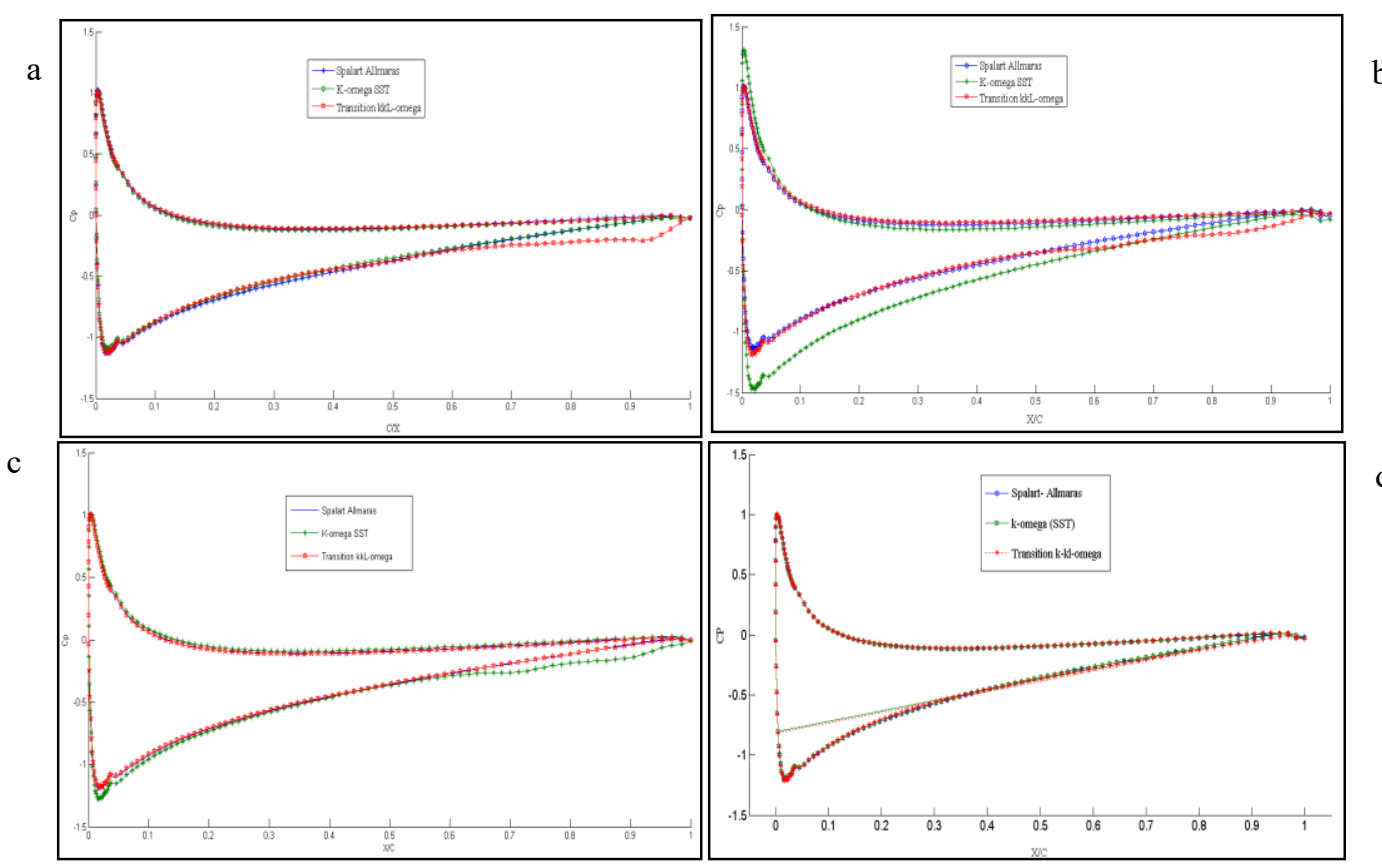

Figure 5. (a) $C_{P}$ Variations vs $x / c$ at $R e=5.10^{4}$, (b) $C_{P}$ Variations vs. $x / c$ at $R e=1.10^{5}$, (c) $C_{P}$ Variations vs $x / c$ at $R e=2.10^{5}$, (d) $C_{P}$ Variations vs $x / c$ at $\operatorname{Re}=1.10^{6}$ from the Current Numerical Work by using Three Different Turbulent Models Simulation Compared with the Numerical Data

Table 1. Comparison between $C_{L}$ and $C_{D}$ for the Best Ratio Given by Predicted and Experimental Results at $R e=5.10^{4}$

\begin{tabular}{lcccc}
\hline & Exp-Data & S-A & K-omega SST & kkL-omega \\
\hline Drag Coefficient & 0.0230 & 0.0291 & 0.0246 & 0.0287 \\
\hline Lift Coefficients & 0.5627 & 0.5372 & 0.4482 & 0.4138 \\
\hline Lift / Drag ratio & 24,4652 & 18,4604 & 18,2195 & 14,4181 \\
\hline
\end{tabular}

Table 2. Comparison between $C_{L}$ and $C_{D}$ for the Best Ratio Given by Predictions and Experimental Results at $\operatorname{Re}=1.10^{5}$

\begin{tabular}{ccccc}
\hline & Exp-Data & S-A & K-omega SST & kkL-omega \\
\hline Drag Coefficient & 0.0195 & 0.0217 & 0.0264 & 0.0313 \\
\hline Lift Coefficients & 0.6862 & 0.5259 & 0.6269 & 0.4554 \\
\hline Lift / Drag ratio & 35,1897 & 24,2350 & 23,7462 & 14,5495 \\
\hline
\end{tabular}


Table 3. Comparison between $C_{L}$ and $C_{D}$ for the Best Ratio Given by Predictions and Experimental Results at $\mathrm{Re}=2.10^{5}$

\begin{tabular}{ccccc}
\hline & Exp-Data & S-A & K-omega SST & kkL-omega \\
\hline Drag Coefficient & 0.0193 & 0.0207 & 0.0236 & 0.0226 \\
\hline Lift Coefficients & 0.8096 & 0.6447 & 0.6115 & 0.7468 \\
\hline Lift / Drag ratio & 41,9481 & 31,1449 & 25,91101 & 33,0442 \\
\hline
\end{tabular}

Table 4. Comparison between $C_{L}$ and $C_{D}$ for the Best Ratio Given by Predictions and Experimental Results at $\mathrm{Re}=1.10^{6}$

\begin{tabular}{ccccc}
\hline & Exp-Data & S-A & K-omega SST & kkL-omega \\
\hline Drag Coefficient & 0.0139 & 0.0194 & 0.0263 & 0.0153 \\
\hline Lift Coefficients & 1.0145 & 0.7974 & 0.6741 & 0.7305 \\
\hline Lift / Drag ratio & 72,9496 & 41,1030 & 25,6311 & 47,7450 \\
\hline
\end{tabular}

Figures 1 to 4 show that the lift and drag coefficient are plotted as functions of the angle of attack, while the Figure 5 shows the pressure coefficient $\left(C_{P}\right)$ which is plotted as a function of chord length. The coefficient behaviors shown in figures are typical. There is a range of angle of attack where the lift coefficient varies linearly. At some points, a maximum value of lift coefficient is reached, where a further increase in angle of attack causes a decrease (sometimes a very rapid decrease) in lift. This referred to as stall. The is a region of lift coefficient where the drag coefficient has its lowest values. Outside this range, it increases, again sometimes rapidly. The pressure coefficients for angle attack of "4" at range of Reynolds number are plotted in Figures 6, 7 and 8. As a results, we can note that with the increasing Reynolds number from $\mathrm{Re}=5.10^{4}$ to $\mathrm{Re}=1.10^{6}$, the pressure difference between upper and lower surface increases. Also with the increasing of angle, pressure difference between upper and lower surface starts to increase.

a

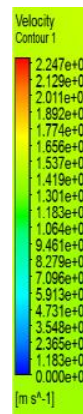

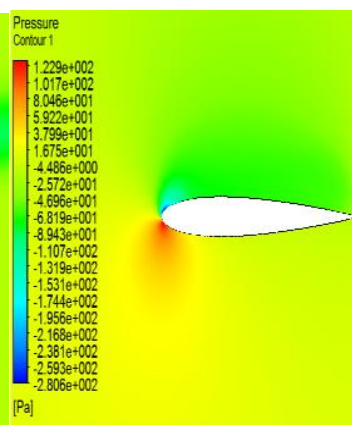

Figure 6. (a) Contours of Velocity, (b) Static Pressure over NACA 0012 Airfoil at Preferred Aerodynamic Ratio Degree of $A O A$ for $R e=1.10^{6}$ by using k-omega SST Turbulence Model 
a

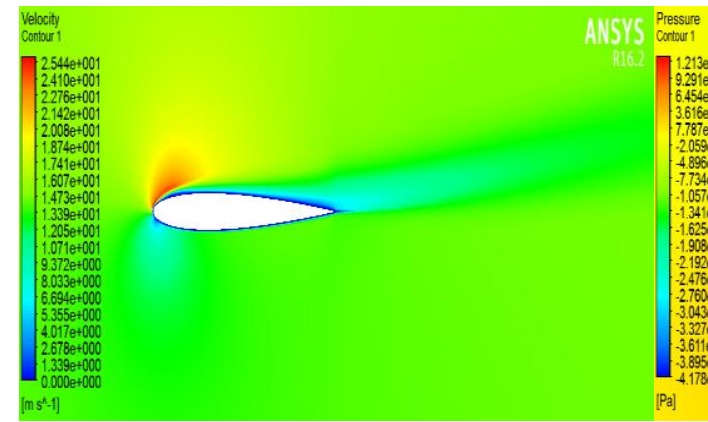

Figure 7. (a) Contours of Velocity, (b) Static Pressure Over NACA 0012 Airfoil at Preferred Aerodynamic Ratio Degree of $A O A$ for $R e=1.10^{6}$ by using Transition kkL-omega Turbulence Model

a

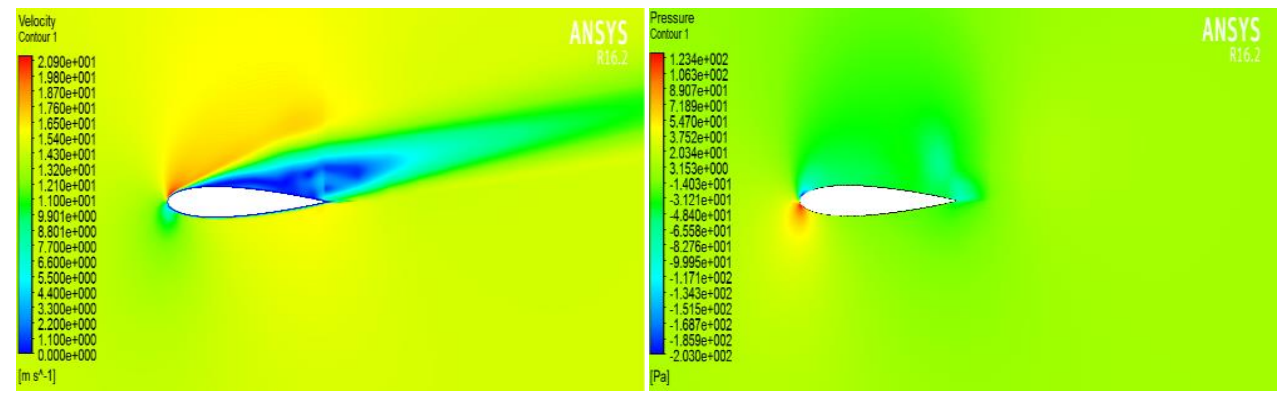

Figure 8. (a) Contours of Velocity, (b) Static Pressure over NACA 0012 Airfoil at Preferred Aerodynamic Ratio Degree of $A O A$ for $R e=1.10^{6}$ by using Spalart-Allmaras Turbulence Model

The change in flow pressure which accompanies separation causes a decrease in lift and an increase in drag. The stability of the laminar boundary layer is affected by the pressure gradient and Reynolds number, low Reynolds numbers and favorable pressure gradient increase stability. From Figures 6 to 8 it can be noted that there is a low pressure region at the top of the airfoil which assists lift as air moves from a region of higher pressure to a region of lower pressure, hence pushing the airfoil upward, normal to the incoming flow stream. On the other hand, the components of the pressure distribution parallel to the incoming flow stream tended to slow the velocity of the incoming flow relative to the airfoil, as do the viscous stresses. The trailing edge stagnation point moved slightly forward on the airfoil at low angles of attack and it jumped rapidly to leading edge at stall angle. A stagnation point is a point in a flow field where the local velocity of the fluid is zero. The upper surface of the airfoil experienced a higher velocity compared to the lower surface. That was expected from the pressure distribution. As the angle of attack increased the upper surface velocity was much higher than the velocity of the lower surface. 
$\mathrm{a}$

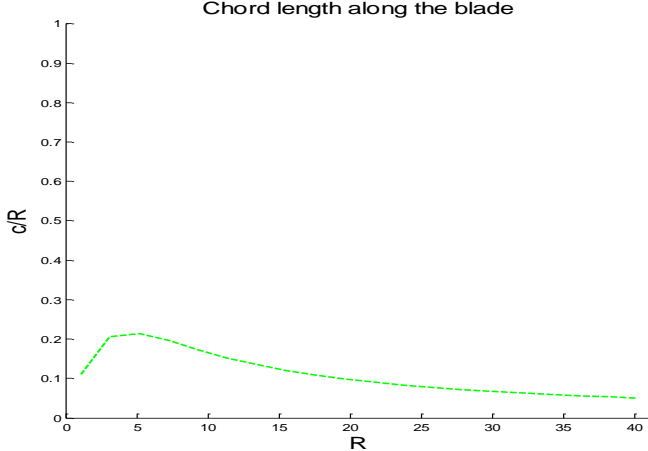

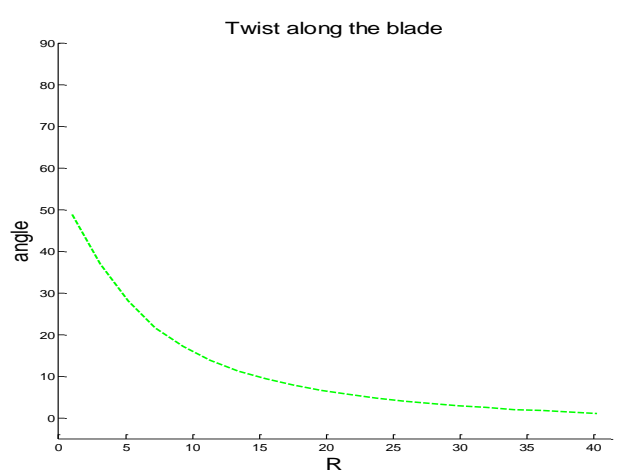

Figure 9. (a) Chord Length as Function of Radius for NACA0012, (b) Twist Angle as Function of Radius for NACA 0012

Figure 9 shows the operating conditions and geometrical properties of the wind turbines with twisted and chord blades using NACA 0012 airfoil section. Based BEM theory it was confirmed that changing of the Reynolds number could have an appreciable effect on the turbine efficiency. The NACA 0012 was also shown to have a higher maximum power output.

\section{Conclusion}

Spalart Allmaras, Transition kkL-omega and the $\mathrm{k}-\omega$ shear stress transport (SST) are used and analyzed in this paper. Among them, the best result for lift Coefficient and the drag coefficient is obtained by Spalart Allamaras model. While, the $\mathrm{k}-\omega$ shear stress transport (SST) turbulence model predicted near stall condition well, whereas two other models were not able to predict it. Also, the experiment can be divided into two regimes: pre-stall and post-stall. In the pre-stall regime different experimental setups give similar drag and lift coefficients for a given angle of attack. This means we can quantitavely compare against the experimental values to validate our simulation methods. In the post-stall regime however, the variation of the lift and drag coefficients for a given angle is large (partly because the stall angle is different), and this does not give a meaningful quantitative validation. Here we compare the post-stall lift (which appears to be roughly similar between the experiments) and check if get a similar low lift over drag in the simulations and experiments. The effects of Reynolds number on the lift coefficient have been investigated. It is realized that by increasing Reynolds number the lift coefficient is increased. The previous analysis can be used in a generalized chord and twist design procedure. The procedure begins with the choice of an airfoil, and then an initial wind blade design is determined using blade element momentum. Additionally, continuation of this analysis would include analyzing different airfoils such as the S-Series airfoils created specifically for Horizontal-Axis Wind Turbines (HAWT).

\section{References}

[1] K. Y. Maalawi, M. A. Badr, "A practical approach for selecting optimum wind rotors", Renewable Energy, vol. 28, (2003), pp. 803-822.

[2] J. Hoffman, C. Johnson, Computational Turbulent Incompressible Flow, Vol. 4 of Applied Mathematics: Body and Soul, Springer, (2007).

[3] A. Spentzos, G. Barakos, K. Badcock, B., Richards, P. Wernert, S. Schreck, M. Raffel, "Investigation of Three-Dimensional Dynamic Stall Using Computational Fluid Dynamics" AIAA Journal, vol. 43, (2005), pp. 1023-1033.

[4] Y. Y. Niu, "Evaluation of Renormalization Group Turbulence Models for Dynamic Stall Simulation", AIAA Journal, vol. 37, (1999), pp. 770-771.

[5] H. Schlicting, K. Gersten, “Boundary Layer Theory”, Springer-Verlag, (2003). 
[6] D. K. Walters, D. Cokljat, "A three-equation eddyviscosity model for reynolds-averaged Navier Stokes simulations of transitional flow". Journal of Fluids Engineering, vol. 130, (2008), pp. 121-401.

[7] B. H. Carmichael, "Low Reynolds Number Airfoil Survey", vol. 1, NASA CR-165803, (1981).

[8] D. Di Pasquale, A. Rona, S. Garrett, “A selective review of transition modelling for CFD". American Institute of Aeronautics and Astronautics, (2009).

[9] F. R. Menter, "Zonal Two Equation k- $\omega$ Turbulence Models for Aerodynamic Flows",AIAA Paper, vol. 93, (1993).

[10] ICEMCFDTM, User's Manual, ICEM CFD System, (1998).

[11] J. L. Tangler, "The Nebulous art of using wind-tunnel airfoil data for predicting rotor Performance", vol. 5, (2002), pp. 245-257. 